\title{
Towards a Self-managing Tool for Optimizing Energy Usage in Buildings
}

\author{
Naveed Arshad, Fahad Javed and Muhammad Dawood Liaqat
}

\begin{abstract}
Smart grid is the next generation of electricity generation, transmission and distribution technology. A major component of smart grid is an overlay communication network for two-way communication between the power providers and the customers. With this feature smart grid provides exciting new ways of energy management and conservation. One of ways to conserve energy using a smart grid is to control and optimize energy usage in buildings. Buildings consume more than one third of the energy produced in the world. Therefore, conserving energy in buildings is cited as the "most important fuel" in energy generation. To this end, we have developed a self-managing approach to optimize energy usage in buildings. We have evaluated our approach using a software tool called Power Conservation Analysis Tool (PCAT). Our initial results using PCAT show upto 38\% savings in the energy bills of customers that could directly translates into reduction in energy production costs for power producers.
\end{abstract}

Key words: optimization, energy usage, self-managing tool

\section{Introduction}

With global warming and impending scarcity of fossil fuel, cleaner sources and better utilization of energy has been considered as a major goal for future technology advancements and research. Energy conservation has been cited as the 'most important fuel' in energy generation [1], [10]. Correspondingly, the smart grid provides a number of ways to conserve energy. One of the ways in which a smart grid provides energy savings is through Advanced Metering Infrastructure (AMI). Through an AMI the energy usage in buildings is monitored through smart meters that are capable of communicating with the smart grid in real time. Electric devices in buildings are connected through a home area network (HAN) using standards such as Zigbee [18], wi-fi, radio or other communication protocols, thus making it possible for

Naveed Arshad, Fahad Javed and Muhammad Dawood Liaqat

Department of Computer Science, LUMS School of Science and Engineering, Lahore, Pakistan, e-mail: naveedarshad, fahadjaved, dawoodliaqat at lums.edu.pk 
all the devices on the network to be managed remotely. The control of these devices through home area networks provide newer ways of conserving energy.

Buildings are the biggest consumers of energy. According to a study, out of all consumers of energy in EU, 37\% of energy is consumed by buildings including both residential and commercial. This is ahead of energy consumption in industrial sector which consumes $28 \%$ and transportation sector which consumes $32 \%$ [13].

One of the ways of energy conservation in buildings is if the consumers control their device usage during peak periods. However, traditionally most energy users in buildings are not interested in manually managing their electric devices to save energy. Rathnayaka and colleagues identified several challenges to of energy management in smart homes [5]. Some of these challenges include a lack of intelligence to handle uncertainty, making passive decisions and others. Research has shown that consumers are willing to engage with the smart grid provided that its interface with the consumers is simple, accessible and in no way interfere with the normal dayto-day life of the consumers [2], [6] . In addition, consumers are also interested in finding that how much can they save in energy bills. This is especially true in case of countries where energy is a scarce and expensive.

Our hypothesis in this research is that if the consumers plan their energy usage according to the supply of energy then just by shifting some energy load to off-peak hours can save energy. To this end, we propose a mechanism where the consumers are not only aware of the energy supply position but their devices are also turned on or off in a self-managed way through employing the priorities and conditions set by the consumers.

To realize this goal, a collaborative technique of energy usage with a constant monitoring of energy devices inputs is required. These inputs are then used in creating a plan to reduce the cost while satisfying conditions and priorities of the customer. To our knowledge, most such systems that provide some sort of interface to the consumer to plan and save power are very few in number and are very rudimentary from a software perspective [8], [14] and [15]. In essence, what is required is a self-managing system that takes user defined goals and produces energy usage plans for the user. Additionally, if the user behavior changes such system must also be able to replan based on the new information.

\section{Scope and Assumptions}

Smart grid vision foresees that the purchasing of electricity will be available directly to the end users rather than through a distribution company. Thus in future, consumers will be charged different prices at different times of the day i.e. hour-ahead pricing, and day-ahead pricing. In this scenarios, where there are time varying prices of electricity, consumers can reduce their electric devices usage expenses by using electric devices at those times when the price of electricity is lower instead of those times when the price of electricity is higher.

Types of Electric Devices

Electric devices have different consumption profiles, therefore, it is imperative that we classify the devices according to their power profiles for controlling them to save energy costs. The devices in almost all types of buildings i.e. residential, 
commercial, etc. can be divided into four categories: 1) Low Power Low Usage (LPL) 2) Low Power High Usage 3) High Power Low Usage (HPL) 4) High Power High Usage (HPH). Since the bulk of energy in any given household is used by the HPL and HPH devices, we believe that if we can manage these devices more intelligently especially in the HPH category then significant cost savings is possible. The devices in this category include air conditioners, water heaters, electric cars, water pumps, etc. Note that our system is mostly focused on residential buildings. For commercial buildings this categorization of devices may not hold.

Device Usage

Amongst electric devices, there are many for which consumer's acceptable usage time ranges can be defined. For example, a consumer might wish to run the water pump for the duration of 45 minutes anywhere between 12:00 AM to 6:00 AM. If one schedule the run of the water pump to a particular 45 minutes time-slot when the price of electricity is the lowest then one can certainly reduce electric power consumption expenses. In such a manner, if one tries to save power consumption expenses on multiple devices in a home on daily basis then the accumulated savings can be significant for the whole billing cycle duration.

There are trade offs between consumer preferences regarding the usage timings of the electric devices and the times when the price of electricity is lower. If electricity consumers use their electric devices at those times when the price of electricity is higher even though the use of those electric devices could be delayed to the times when the price of electricity will be lower, then certainly the consumers will be having relatively higher electric power consumption expenses. So, if we have consumer preferences regarding the electric devices usage timings then we can reduce the electric devices usage expenses by optimally switching electric devices ON/OFF in a time varying electricity prices-aware manner while satisfying the preferences, at the same time. Given a set of electric devices, user preferences regarding the usage timings for each of the devices and the time varying prices of electricity, we describe an approach to develop electric devices usage plan for consumer.

\section{Approach}

In our approach we assume that "hour-ahead" pricing is available from the energy market. This means that the energy prices are known one hour before.

Priorities and Constraints Specification

A typical building may have a number of devices on the HAN. PCAT has an editable list of devices present on the HAN. Using PCAT a consumer selects the devices that are flexible in their usage. The consumer provides a set of priorities and constraints for each device. These are specified at a high-level by using a very simple user interface. Each device can have multiple set of priorities and goals. For this paper, we assume that these goals are not conflicting with one another.

Other than providing this the consumer also specifies the maximum total cost of electricity that he or she is willing to pay at the end of a billing cycle. This is especially beneficial for usage in countries where the price of per unit electricity increases with more usage. These pieces of information are used by our optimiza- 
tion algorithm to generate a plan for energy management and consequently energy savings.

Optimization Strategy

We consider a set of electric devices for which a home consumer wish to generate the optimal usage plan such that the power consumption expenditure is minimized by taking advantage of the time varying prices of electricity. For each of the devices to be controlled on the HAN, average hourly energy consumption profile is available. Each device can have multiple consumer preferences regarding its usage timing limits and the duration to keep the device in a certain state. For simplicity purposes, in this paper, we have only considered devices whose states can be turned on or off only. However, in reality multiple ways of energy conservation are possible. This includes controlling the thermostat, reducing the voltage supplied to a certain device, providing energy from multiple source i.e. a solar cell and the electricity from the power company, etc.

We would like to stress here that our optimization strategy does not turn the devices on or off without implicit permission by the user. The devices can only be turned on or off in the given time windows specified by the user.

Let us discuss the energy conservation strategy for an electric dishwasher. An automatic electric dishwasher can have a preference that it should be scheduled to run anywhere between 9:30 AM to 12:30 PM for 45 minutes. Similarly for the same dishwasher another preference can be that it should be scheduled to run anywhere between 4:00 PM to 7:00 PM for 45 minutes. Consider the later preference, we call 4:00 PM as the lower time limit and 7:00 PM as the upper time limit during which the dishwasher must be switched on. Our objective is to find that particular 45 minutes time slot anywhere between the lower and upper time limits at which the price of electricity is the minimum.

However, the energy price is only known before the start of the hour. Therefore, to develop a 24 hour plan ahead of of its start, we use historical average of prices. If the price of electricity for each hour is between a bound of the actual electricity prices then the originally created plan will be followed. These bounds are determined by the maximum bill amount a consumer is willing to pay. However, if the difference of the actual energy price and expected energy price is more than a given bound then a replanning process will take place to calculate another plan based on the new information about the energy prices.

\section{Optimization Algorithm}

We have used the concept of timing windows to implement our algorithm. The detailed implementation is described as follows:

Let $D=\left\{d_{1}, \ldots, d_{n}\right\}$ is the set of $n$ electric devices. The electric power consumption of the $n$ devices is represented by the set $K=\left\{k_{1}, \ldots, k_{n}\right\}$ where each element $k_{i}$ denotes the electric power consumption for the device $d_{i} \in D$. The preferences for an electric device $d_{i} \in D$ are represented using the set $P^{i}$ where each element $p_{j}^{i}$ is itself a tuple defined as $p_{j}^{i}=<l_{p_{j}^{i}}, u_{p_{j}^{i}}, o_{p_{j}^{i}}>$. Among the elements of the tuple, $l_{p_{j}^{i}}$ is the lower time limit, $u_{p_{j}^{i}}$ is the upper time limit and $o_{p_{j}^{i}}$ is the ON duration for $j^{\text {th }}$ preference of $i^{\text {th }}$ device. Time varying prices of electricity are given as a set $C=\left\{c_{1}, \ldots, c_{m}\right\}$ where each element $c_{t}$ denotes the price of per 
unit electricity for the time duration between time instances $t$ (inclusive) and $t+1$ (exclusive).

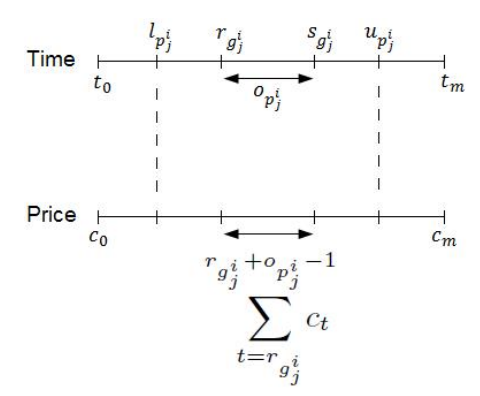

Fig. 1: Finding optimum switch-ON time

Our objective is to find a set $G^{i}$ corresponding to each set of preferences $P^{i}$ such that for each preference $p_{j}^{i} \in P^{i}$ the set $G^{i}$ has one element $g_{j}^{i}$ which is an ordered pair of the form $g_{j}^{i}=<r_{g_{j}^{i}}, s_{g_{j}^{i}}>$. Inside the ordered pair, $r_{g_{j}^{i}}$ and $s_{g_{j}^{i}}$ denote the most suitable times to turn ON and OFF the $i^{\text {th }}$ device, respectively for satisfying $j^{\text {th }}$ preference. Each $s_{g_{j}^{i}}$ can be calculated as $s_{g_{j}^{i}}=r_{g_{j}^{i}}+o_{p_{j}^{i}}$ that is, the time at which to turn ON the device plus the duration to keep it ON. The overall electricity consumption expense of the devices should be the minimum if the generated plan consisting of all $G^{i}$ is followed.

We have to find each $r_{g_{j}^{i}}$ for which $f\left(r_{g_{j}^{i}}\right)$ is the minimum where $f\left(r_{g_{j}^{i}}\right)$ denotes the expense of switching $\mathrm{ON}$ the $i^{\text {th }}$ device at the time $r_{g_{j}^{i}}$ and keeping it $\mathrm{ON}$ for the duration determined by $o_{p_{j}^{i}}$. This is according to $j^{\text {th }}$ preference of the same device. The function $f\left(r_{g_{j}^{i}}\right)$ can be defined iteratively as:

$$
f\left(r_{g_{j}^{i}}\right)=\sum_{t=r_{g_{j}^{i}}}^{r_{g_{j}^{i}}+o_{p_{j}^{i}}-1} c_{t}
$$

The function $f\left(r_{g_{j}^{i}}\right)$ can also be defined recursively. For recursive case when $r_{g_{j}^{i}}>$ $l_{p_{j}^{i}}$ then

$$
f\left(r_{g_{j}^{i}}\right)=f\left(r_{g_{j}^{i}}-1\right)-c_{\left(r_{g_{j}^{i}}-1\right)}+c_{\left(r_{g_{j}^{i}}+o_{p_{j}^{i}}-1\right)}
$$

For base case when $r_{g_{j}^{i}}=l_{p_{j}^{i}}$ then again

$$
f\left(r_{g_{j}^{i}}\right)=\sum_{t=r_{g_{j}^{i}}}^{r_{g_{j}^{i}}+o_{p_{j}^{i}}-1} c_{t}
$$


Recursive definition is cheaper in terms of calculation because it avoids repetitive calculations. In either of iterative or recursive case, we have to minimize $f\left(r_{g_{j}^{i}}\right)$ subject to following constraints:

$$
\begin{gathered}
r_{g_{j}^{i}} \geq l_{p_{j}^{i}} \\
r_{g_{j}^{i}} \leq u_{p_{j}^{i}}-o_{p_{j}^{i}} \\
\forall i, j
\end{gathered}
$$

Figure 1 shows how the time window determined by each $<r_{g_{j}^{i}}, s_{g_{j}^{i}}>$ pair can slide within the time limits determined by the preference $p_{j}^{i}$. We have to find those points $r_{g_{j}^{i}}$ and $s_{g_{j}^{i}}$ on the timeline where the expenditure value of the sliding window comes out to be the minimum.

\section{Architecture and implementation}

PCAT is a web application developed for displaying electricity consumption statistics and graphs to electricity consumers. PCAT is based on the model that electricity consumers have smart electricity meters installed at their homes. These meters are capable of transmitting periodic electricity consumption statistics of the consumption database of the PCAT. Consumers can then logon to PCAT to view their power consumption statistics and various types of charts on daily, weekly, monthly, yearly and on billing cycle duration basis. Consumers can check their tariff based on their chosen tariff package and the consumption made during the billing cycle. Consumers are also able to plan the consumption of their electric devices during the billing cycle while staying within a consumer chosen desired bill amount.

The most interesting component of PCAT is the Optimization Manager (OM). The OM interfaces with the HAN to access electric device information and updates its database accordingly. OM also interfaces with the smart grid interface to get upto date energy prices and also to get information from the AMI. Based on the customer preferences, it invokes a planning module to generate a plan for energy consumption based on historical price data. The planning module gets the estimated price information from an estimation module that uses a moving averages algorithm to calculate the estimated electricity prices. The OM also triggers a replanning process, if the energy prices are not in between certain bounds. The replanning is performed for the rest of the hours in a 24 hour cycle.

Other than the OM, the layers architecture has a Presentation Layer (PL) and a Data Layer (DL). The PL is used as an interface with the consumer and the DL is used to connect with the database that holds all the necessary data to run the application.

PCAT is developed as a web application using Microsoft ASP.NET, Visual Cl\# and Microsoft SQL Server.

\section{Evaluation}

This section reports preliminary experimental results using PCAT for optimizing power utilization in a typical household scenario. 


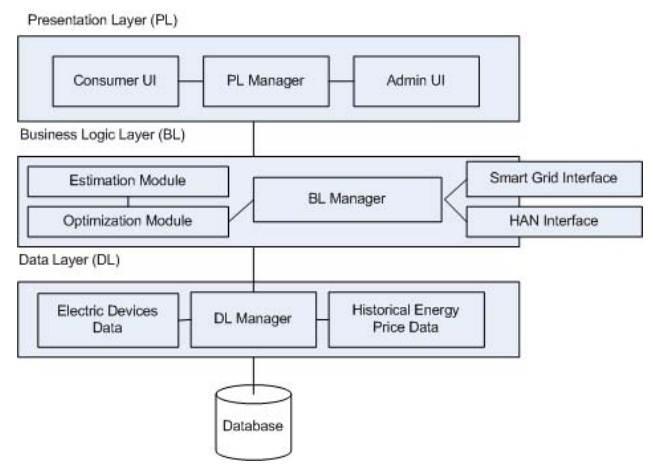

Fig. 2: PCAT Architecture

We run our experiments using profiles of electric devices in an average-sized house. This include air conditioners, washing machine, dishwasher, water pump, etc. In all, we considered 7 different devices in our sample household. Table 1 lists the devices and respective energy profiles. We considered the general use of these devices and set the preferences accordingly. The 15 preferences we used for experiments are shown in figure 3. In this figure the preferred time of usage is shown as an interval on the time line. Some devices, such as ACs are used multiple times in a day whereas some devices are only used once. It can be noted that different types of devices are being used at night so that the services they provide are available in the morning. This include the water pump, dish washer and battery charging for electric vehicles. Our third source of data is the power tariff that is applicable for a given 30 minute period. We used the data from the New York Independent System Operator's ${ }^{1}$ website for first 7 days in the month of September 2009.

Table 1: Devices

\begin{tabular}{|c|c|c|}
\hline $\begin{array}{c}\text { Device } \\
\text { ID }\end{array}$ & $\begin{array}{c}\text { Device } \\
\text { Name }\end{array}$ & $\begin{array}{c}\text { Average Hourly } \\
\text { Consumption } \\
(\mathrm{kWh})\end{array}$ \\
\hline 1 & Air Conditioner 1 & 2 \\
\hline 2 & Air Conditioner 2 & 1.5 \\
\hline 3 & Air Conditioner 3 & 2 \\
\hline 4 & Washing Machine & 1.5 \\
\hline 5 & Dishwasher & 1 \\
\hline 6 & Electric Car Battery Charger & 2.5 \\
\hline 7 & Water Pump & 1.5 \\
\hline
\end{tabular}

\footnotetext{
${ }^{1}$ http://www.nyiso.com/
} 


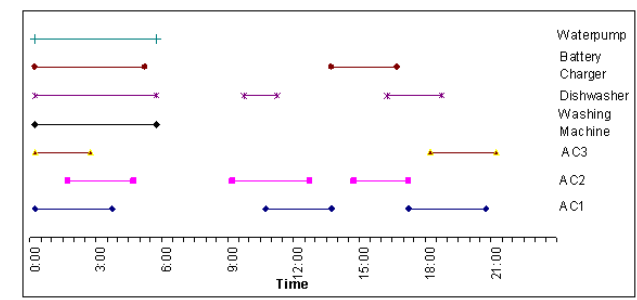

Fig. 3: Preferences for the 7 devices in the household

A user oblivious to the price variation will use the device any time within the limits of his preferences. Using this hypothesis we run two types of simulation. For both of these simulations we fixed the amount of energy that is used the house.

Our first simulation uses a pseudo-random selection of device usage. This is to simulate a typical energy usage behavior in a household. The second simulation is performed using the Optimization Manager (OM) of PCAT. We executed multiple runs of both the scenarios and averaged out the results. The results of this comparison are shown in figure 4.

As we can see the optimized plan costs less than the typical scenario on each of the seven days. The typical plan is at an average $19 \%$ more costly than the optimal plan and on some days, such as on Day 6 it is more than $38 \%$ more costly than the optimized plan. It was noted during the multiple runs of the typical plan that not even once did the typical plan match the performance of the optimized plan.

The benefits from optimization is due to the variations in price in a day. We observed that on a given day, the price of power varied from $\$ 356 / \mathrm{MWh}$ to $\$ 5.64 / \mathrm{MWh}$ with standard deviation as high as $\$ 65$ over a period of a single day. This variation in price is used by our optimizer to deliver the results we see in our experiments.

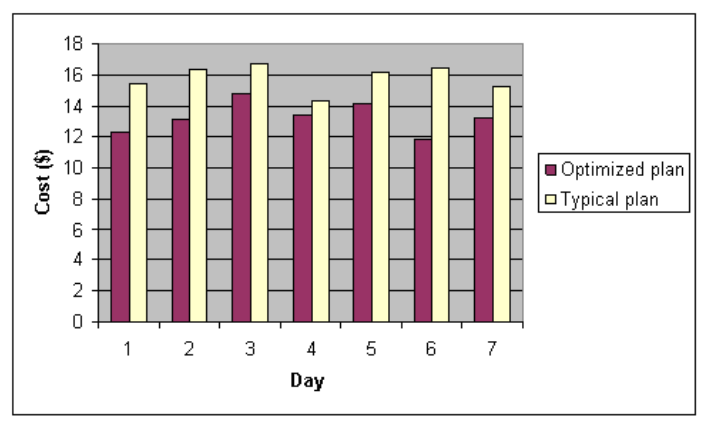

Fig. 4: Comparison of optimized plan and average of typical plan oblivious of price 


\section{Related Work}

A variety of self-managing power management techniques have been developed which provide power management support to large scale computing systems such as server farms etc. Works such as by Milenkovic and colleagues use the powerawareness in management of cloud data centers [11]. Nathuji and colleagues proposed mechanisms to manage data centers by using the heterogeneity of machines [12] and and Zhu and colleagues proposed a framework for hierarchical optimization scheme for managing large scale, distributed data centers [17]. Works of Ha and Abras provide methods to optimize power consumption in smart-homes [3,9] . However, these systems are made with the scope that the user does not have the capability to provide the preferences of usage thus these systems have a heavy component to predict the usage of electricity and to calculate the effectiveness of plan through secondary measures. Through its user interface, PCAT is able to bypass these procedures by gathering the usage and effectiveness of plan data directly from the user. This not only simplifies the optimization methods but also make it efficient enough in terms of speed for large scale deployment.

There are some supporting works that can better serve users in conjunction with PCAT. These include Genio by Gï̈œrate et al. which uses Ambient Intelligence for human interaction with home environment where user can control home appliances by talking in a natural way [7]. Alkar et al. [4] developed a low cost, secure Internet based wireless system for home automation which can control a wide variety of devices. And Yuksekkaya et al. [16] developed a low cost, user friendly, wireless interactive home automation system which can be controlled by GSM, Internet and speech.

\section{Conclusions and Future Work}

In this paper, we have showed that home electricity consumers can save money on electricity bills by taking advantage of the time varying prices of electricity together with saving electricity production costs. The basic rational is that when we have time varying prices of electricity available then we can reduce electric devices usage expenses by using electric devices at those times when the price of electricity is lower.

Many future opportunities ahead in the area of smart grid for the research community. Other than device on/off there are various ways of intelligent energy usage. For example, through reducing the voltage to a device, through adding multiple sources of energy i.e. solar panel on the rooftop etc. Using these various ways of conserving energy has a potential for very complex, interesting and meaningful problems for the research community. Moreover, using an extension of HAN the self-managing system could also be applied to a Neighborhood Area Network (NAN) where a neighborhood becomes an energy island and has its own ways of producing and consuming electricity.

We further plan to extend PCAT to handle some of the challenges of energy management in smart homes as identified by Rathnayaka and colleagues [5]. These include handling conflicting multiple user priorities, multiuser buildings and so on and so forth. 


\section{References}

1. Energy Use in the New Millennium - Trends in IEA Countries. International Energy Agency (2007)

2. The smart grid: An introduction (2009). US Department of Energy

3. Abras, S., Pesty, S., Ploix, S., Jacomino, M.: An anticipation mechanism for power management in a smart home using multi-agent systems. In: Information and Communication Technologies: From Theory to Applications, 2008. ICTTA 2008. 3rd International Conference on, pp. 1-6 (2008). DOI 10.1109/ICTTA.2008.4530305

4. Alkar, A., Buhur, U.: An internet based wireless home automation system for multifunctional devices. Consumer Electronics, IEEE Transactions on 51(4), 1169-1174 (2005). DOI 10.1109/TCE.2005.1561840

5. Dinusha Rathnayaka, A.J., Potdar, V.M., Kuruppu, S.J.: Energy resource management in smart home: State of the art and challenges ahead. In: N. MSirdi, A. Namaane, R.J. Howlett, L.C. Jain, R.J. Howlett, L.C. Jain (eds.) Sustainability in Energy and Buildings, Smart Innovation, Systems and Technologies, vol. 12, pp. 403-411. Springer Berlin Heidelberg (2012)

6. Feinberg, R.: Achieving customer acceptance of the smart grid (2009). The Intelligent Project

7. Gárate, A., Herrasti, N., López, A.: Genio: an ambient intelligence application in home automation and entertainment environment. In: sOc-EUSAI '05: Proceedings of the 2005 joint conference on Smart objects and ambient intelligence, pp. 241-245. ACM, New York, NY, USA (2005). DOI http://doi.acm.org/10.1145/1107548.1107609

8. Gill, K., Yang, S.H.Y.S.H., Yao, F.Y.F., Lu, X.L.X.: A zigbee-based home automation system (2009). URL http://ieeexplore.ieee.org/lpdocs/epic03/wrapper.htm?arnumber=5174403

9. Ha, D.L., de Lamotte, F., Huynh, Q.H.: Real-time dynamic multilevel optimization for demand-side load management. In: Industrial Engineering and Engineering Management, 2007 IEEE International Conference on, pp. 945-949 (2007). DOI 10.1109/IEEM.2007.4419331

10. Javed, F., Arshad, N.: A penny saved is a penny earned: Applying optimization techniques to power management. In: 16th IEEE International Conference on the Engineering of ComputerBased Systems (ECBS 2009), 13-16 April 2009, San Francisco, CA, USA (2009)

11. Milenkovic, M., Castro-Leon, E., Blakley, J.R.: Power-aware management in cloud data centers. In: CloudCom '09: Proceedings of the 1st International Conference on Cloud Computing, pp. 668-673. Springer-Verlag, Berlin, Heidelberg (2009)

12. Nathuji, R., Isci, C., Gorbatov, E.: Exploiting platform heterogeneity for power efficient data centers. Autonomic Computing, 2007. ICAC '07. Fourth International Conference on pp. 5-5 (11-15 June 2007). DOI 10.1109/ICAC.2007.16

13. Pérez-Lombard, L., Ortiz, J., Pout, C.: A review on buildings energy consumption information. Energy and Buildings 40(3), 394 - 398 (2008). DOI DOI: 10.1016/j.enbuild.2007.03.007. URL http://www.sciencedirect.com/science/article/B6V2V4N8BMX9-2/2/5d59d6827f1f8d36a2a6480a0c95650e

14. Wallin, F., Bartusch, C., Thorin, E., Bdckstrom, T., Dahlquist, E.: The use of automatic meter readings for a demand-based tariff. pp. 1-6 (2005). DOI 10.1109/TDC.2005.1547125

15. Wallin, F., Dotzauer, E., Thorin, E., Dahlquist, E.: Automatic meter reading provides opportunities for new prognosis and simulation methods. pp. 2006-2011 (2007). DOI 10.1109/PCT.2007.4538626

16. Yuksekkaya, B., Kayalar, A., Tosun, M., Ozcan, M., Alkar, A.: A gsm, internet and speech controlled wireless interactive home automation system. Consumer Electronics, IEEE Transactions on 52(3), 837-843 (2006). DOI 10.1109/TCE.2006.1706478

17. Zhu, X., Young, D., Watson, B., Wang, Z., Rolia, J., Singhal, S., McKee, B., Hyser, C., Gmach, D., Gardner, R., Christian, T., Cherkasova, L.: 1000 islands: Integratedcapacity and workload management for the next generation data center. In: Autonomic Computing, 2008. ICAC ' 08 . International Conference on, pp. 172-181 (2008). DOI 10.1109/ICAC.2008.32

18. Zigbee, http://www.zigbee.org/, accessed 20th march, 2012 